\title{
El ramipril reduce la mortalidad en la insuficiencia cardiaca post-infarto
}

Follow-up study of patients randomly allocated ramipril or placebo for heart failure after acute myocardial infarction:

AIRE Extension (AIREX) Study.

Hall AS, Murray GD, Ball SG on behalf of the Airex Study Investigators. Lancet 1997, 349: 1493-97.

\section{Objetivo}

Evaluar la eficacia a largo plazo del ramipril.

\section{Diseño}

Ensayo multicéntrico, randomizado, doble ciego y controlado con placebo.

\section{Lugar}

Participaron 30 centros del Reino Unido.

\section{Pacientes}

Se incluyeron 603 pacientes con evidencias de insuficiencia cardíaca post-infarto agudo de miocardio (post-IAM), pero sin inestabilidad clínica ni clara indicación o contraindicación de Inhibidores de la Enzima Convertidora de Angiotensina (IECA).

\section{Intervención}

De 2 a 9 días post-IAM se titulaba la dosis de ramipril hasta alcanzar, de ser posible, $5 \mathrm{mg} / 12 \mathrm{Hs}$. La duración de tratamiento fue $13.4 \mathrm{me}-$ ses para placebo y 12.4 meses para ramipril luego de lo cual los médicos tratantes decidían la terapéutica subsiguiente (no consignada), desconociendo el grupo al que había sido asignado su paciente. El seguimiento medio fue de 59 meses (mínimo 42).
Medición de resultados principales

Mortalidad por todas las causas analizada por intención de tratar*.

\section{Resultados principales}

La mortalidad fue de $38.9 \%$ entre los 301 pacientes asignados a placebo y de $27.5 \%$ entre los 302 asignados a ramipril: reducción de riesgo absoluto (RRA) de $11.4 \%$ y relativo (RRR) de $36 \%$ (95\% IC 15-52\%, $\mathrm{p}=0.002$ ).

\section{Conclusiones}

El ramipril reduce considerablemente la mortalidad, por al menos 5 años, en pacientes con insuficiencia cardíaca post-IAM.

\section{COMENTARIO}

Varios puntos refuerzan los hallazgos del estudio. Primero, pudo verificarse la sobrevida de todos los pacientes (el seguimiento fue del $100 \%)$. Segundo, en el grupo placebo, en el que más pacientes desarrollaron Insuficiencia Cardíaca, hubo más pacientes que salieron del estudio para recibir IECA y posiblemente muchos pacientes del mismo grupo también los hayan recibido durante el seguimiento extendido. Sin embargo, a pesar de que esto podría disminuir los efectos beneficiosos del ramipril, no se anuló el efecto en el análisis por intención de tratar*. Mas aun, este beneficio que comienza a ser significativo al cumplirse el mes, sigue acrecentándose hasta un año después de suspendido el estudio, y se mantiene estable entre los 2 a los 5 años, a pesar de que el poder* para detectar diferencias luego de los.2 años es bajo. Esto podría significar que los sobrevivientes del grupo asignado a placebo tendrían un perfil de riesgo similar al del grupo ramipril a partir de los dos años, o que estos pacientes tuvieran acceso irrestricto a los IECA una vez finalizado el estudio. En base a los resultados de mortalidad obtenidos (RRA de $11.4 \%$ y RRR de $36 \%$ [IC 95\% $15-52 \%]$ ), cada 9 pacientes tratados con ramipril durante un año se salvaría una vida en 5 años (Número Necesario para Tratar o NNT=9; IC95\% 5-17). La magnitud del efecto es similar a la del bypass corona- rio en lesión del tronco de la coronaria izquierda (NNT=6 pacientes para lograr igual sobrevida). Un amplio meta-análisis (1) de 9 ensayos randomizados sobre IECA en el post-IAM (>100.000 pacientes) documentó una RRA en la mortalidad de $0.46 \%$ y RRR de $6.5 \%$ (IC95\% 1.9 $11.1 \%$ ), o lo que es lo mismo, un NNT de 217 para diferentes IECA, indicando un "efecto benéfico de clase". Sin embargo estos estudios deben dividirse en dos grupos. Uno, en que los IECA se indicaban a pacientes no seleccionados dentro de las $48 \mathrm{Hs}$. del IAM (CONSENSUS II, GISSI-3, GISSI-4, CCS-1) y tratamiento de sólo 4 a 6 semanas en los tres últimos, encontrando modestos o nulos beneficios. Otro, en que se indicaban luego de $48 \mathrm{Hs}$. y/o en pacientes con disfunción sistólica (SAVE, TRACE), IC (AIRE) o IAM anterior (SMILE) con seguimiento de 1 a 4 años y significativas reducciones de mortalidad de $19-29 \%$ (NNT=17-25). El AIREX refuerza los resultados de este grupo, encontrando aún más beneficio al extender el seguimiento (NNT=9). Lamentablemente los IECA son subutilizados (2) y es imperativo que resultados tan favorables puedan finalmente trasladarse a la práctica. En particular, los hallazgos a largo plazo de este estudio, avalan la recomendación de continuar indefinidamente con los IECA, en pacientes con Insuficiencia Cardíaca post-IAM, excepto que no sean tolerados.

*Ver Glosario

\section{Dr. Agustín Ciapponi}

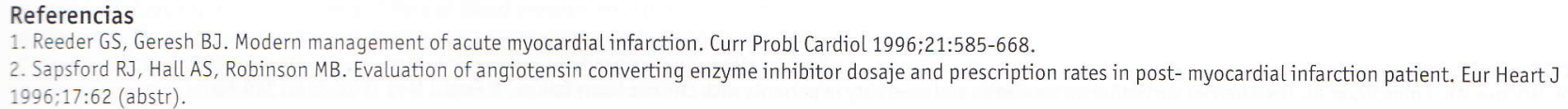

